\title{
Design and Implementation of GA Filter Algorithm for Baro-inertial Altitude Error Compensation
}

\author{
Jafar Keighobadi $^{1}$, Hossein Nourmohammadi ${ }^{2}$ and Sadra Rafatnia ${ }^{3}$
}

\begin{abstract}
Providing accurate altitude plays a key role in the long-term performance and reliability of inertial Navigation systems (INSs). In low-cost MEMS-grade INSs, failure to compensate vertical channel instability errors could result in exponentially divergence of computed altitude. Therefore, an integrated baroinertial altimeter comprising of a damping loop and optimal state estimation mechanizations leads to compensated altitude error in vertical channel of navigation systems. In this paper, considering different environmental conditions in the form of standard and nonstandard atmosphere models and using a MEMS altimeter, the barometric altitude is accurately computed. Then, the genetic algorithm (GA) is utilized for integrating the computed barometric altitude with that of the vertical channel of MEMS-grade INS. Vehicular test has been carried out to show the long-term performance of the proposed Baro-inertial integrated system.
\end{abstract}

Keywords - Integrated Baro-inertial altimeter, Barometric altitude, Vertical channel instability, Genetic algorithm.

\section{INTRODUCTION}

A ided altitude measurement is one of the most important inputs for damping the vertical channel instability of inertial navigation system (INS). In common INS, the barometric pressure is utilized to stabilize and control the divergence of altitude/height and down-ward velocity. Using relatively stable measurements of barometric pressure sensor and suitable atmosphere models, the accurate altitude above sea level can be obtained. According to standard atmosphere models, barometric altitude is simply computed based on the static pressure. Since, the true sea level temperature and pressure are not fixed values, their deviation from the assumed fixed values by the standard atmosphere model results in large altitude errors. Even though the data are established before take-off, climbing or diving, the non-standard atmosphere can result in up to $8 \%$ error of the height rate together with corresponding error in the height [1].

In order to avoid the above mentioned drawback, a nonstandard atmosphere model is considered in the paper. In this

Jafar Keighobadi ${ }^{1}$ is with the University of Tabriz, Tabriz, CO 5166614766 Iran

Hossein Nourmohammadi ${ }^{2}$, is with the University of Tabriz, Tabriz, Iran.

Sadra Rafatnia ${ }^{3}$, was with University of Tabriz, Tabriz, Iran. He is now with the Department of Mechanical Engineering, Sahand University of Technology, Tabriz, Iran method, the barometric altitude error which arises from nonstandard atmosphere conditions is compensated.

Vertical channel damping loop and optimal state estimation algorithms based on Kalman filter (KF) are two main methodologies that have been addressed in literatures concerned to developing integrated baro-inertial altimeters. The vertical channel damping loop is a feedback control system in which a PI feedback of the altitude error between INS and barometer outputs is utilized to compensate the vertical channel errors [2], [3]. Kalman filter as a recursive algorithm to provide optimal estimation of state vector of a linear dynamic system is generalized to its extended type (EKF) dealing with nonlinear systems [4]-[6]. Using stochastic optimal control approach, Widnall and Sinha determined the optimal gains of the baro-inertial vertical channel [7]. Ausman et al. [8] developed a KF algorithm for vertical channel mechanization based on a linear error model including calibration scale factor of altitude. Seo et al. [9] proposed a KF algorithm for estimation and compensation of error dynamics of vertical channel in which GPS output data are used as the measurement vector. Using sigma point hypotheses a new filter design was presented for identification of barometric aiding sensor of the vertical channel damping loop [10]. Different error sources in differential barometry for personal application have been studied in [11]. A stochastic model has been developed in [12] to explore main statistical properties of the barometric altimeter's noises.

In this paper, based on genetic algorithm (GA) as a global optimization method, a new filtering algorithm is designed for the purpose of barometric altitude error compensation in the damping loop of the MEMS INS vertical channel. GA is a stochastic global search method inspired by the process of natural evolution [13], [14]. Compared to the classic KF, the superior estimation of the integrated baro-inertial altimeter is obtained owing to the GA filter attributes as follows.

1) The GA filter is initialized with no knowledge of correct solution and entirely depends on the responses by the environment and the evolution operators (crossover and mutation), and thus reaches to the best solution.

2) By starting at several independent points and searching in parallel, the algorithm avoids local minimal and even converging to suboptimal solutions.

3) The optimal solution of the proposed algorithm is not limited to zero-mean Gaussian noise processes with known 
covariance matrices. The filter performance is not involved with substantial restriction occurs by using classic KF.

\section{VERTICAL CHANNEL MECHANIZATION}

This section deals with the formulation of true barometric altitude model in non-standard atmosphere conditions and developing vertical channel damping loop. The barometric altimeter is affected in a large degree by the atmosphere physics. So, it must be calibrated. In the standard atmosphere, the barometric altitude is obtained as follows [1].

$H_{p}=\frac{T_{0}}{L}\left[\left(\frac{P_{s}}{P_{0}}\right)^{-\frac{L R}{g}}-1\right]+H_{0}$

where, the temperature and the pressure at sea level are specified by $T_{0}$ and $P_{0}$, respectively. In standard atmosphere these variables are assumed to be $288.15\left({ }^{\circ} \mathrm{K}\right)$, $101.325(k P a)$. L, $\mathrm{R}$, and $\mathrm{g}$ are the constant lapse rate, universal gas constant, and gravity constant, respectively. $H_{0}$ is taken 0 for sea level data, and $P_{s}$ stands for the pressure measured by the barometer. For non-standard atmosphere, the constant lapse rate assumption remains valid [15]. However, the temperature and the pressure assumptions cannot be guaranteed as a real atmosphere system. Applying scale factor, $\mathrm{s}$ and bias, $\mathrm{b}$ as calibration parameters, the barometric altitude compensation is achieved by the following equation of nonstandard atmosphere.

$$
H \square H_{P}+s\left(H_{P}-H_{0}\right)+b
$$

where, $H$ is the calibrated barometric altitude under nonstandard atmosphere condition. The scale factor and the bias parameters are defined as follows.

$$
\begin{aligned}
& s=\frac{\Delta T}{T_{0}} \\
& b=\frac{R T_{0}}{g}\left(\frac{\Delta P}{P_{0}}\right)
\end{aligned}
$$

In (3) and (4), $\Delta T$ and $\Delta P$ denote the deviation of true temperature and true pressure at sea level from the corresponding fixed values of standard condition, respectively. To compute the true values of local sea level temperature and pressure around tests region, the following model is used.

$$
\begin{aligned}
& T_{t}=T_{s}+L H_{P} \\
& P_{t}=P_{s}+\rho g H_{p}
\end{aligned}
$$

where, $T_{t}$ and $P_{t}$ represent the true temperature and the pressure at sea level, respectively. $T_{s}$ and $P_{s}$ are the measured values by sensors.

In many positioning and navigation applications accurate altitude information is required. The main goal of the vertical channel mechanization is to minimize the errors of altitude and down-ward vertical velocity. Through damping loop, the vertical channel data of INS is integrated with aiding barometric system known here as Air-data. The feedback block diagram of damping loop in Fig. 1 shows how the computed data of INS vertical channel are stabilized.

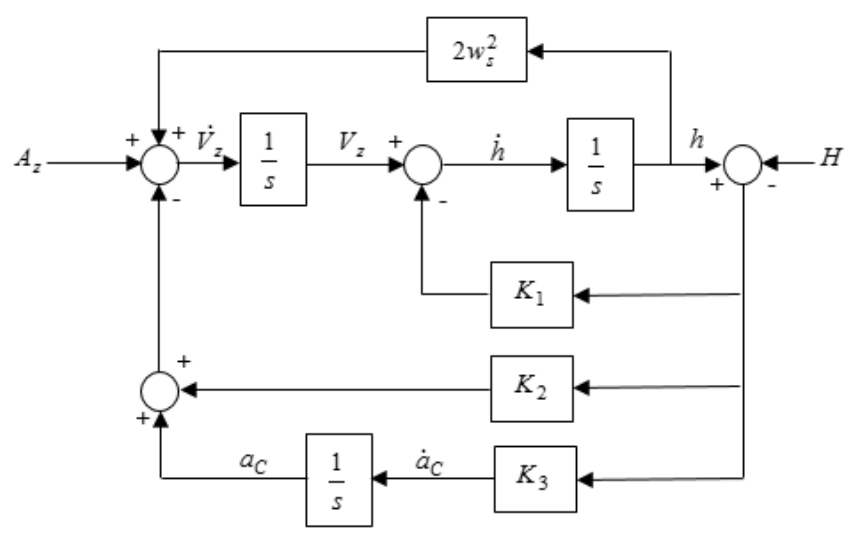

Fig.1 Block diagram of vertical channel damping loop.

The corresponding dynamical equation of the damping loop can be written as follows.

$\dot{h}=V_{Z}-K_{1}(h-H)$

$\dot{V}_{Z}=A_{Z}-a_{C}+2 w_{s}^{2} h-K_{2}(h-H)$

$\dot{a}_{C}=K_{3}(h-H)$

where, $V_{Z}, h$ and $H$ stand for vertical velocity, true height and calibrated barometric altitude of non-standard atmosphere condition, respectively. $A_{Z}$ denotes the measured acceleration along vertical channel. The parameters, $a_{C}$ and $w_{s}$ are the outputs of compensator and Schuler frequency, respectively. The feedback gains, $K_{1}, K_{2}$ and $K_{3}$ should be determined in such a way that leads to minimized error between the estimated and the reference values of vertical velocity. GA based computation algorithm for optimal values of feedback gains is explained in complete details in the following section.

\section{ERROR COMPENSATION BY GA METHOD}

In this section, a new binary GA method is proposed. As a matter of fact, GPS and barometer are used as aiding systems of the integrated INS to estimate and compensate its vertical channel errors. The vertical velocity component of GPS system, $V_{G}^{o b s}$ is considered as the reference signal and the value computed by (6), $V_{Z}^{a}$ is considered as the estimated value. The mismatch between the $N$ number of reference and estimated values is designated based on the following cost function.

$J_{O} \equiv \sum\left(V_{G i}^{o b s}-V_{Z i}^{a}\right)^{2} \quad i=1,2, \ldots, N$

where, the summation extends over the observation grid points by the GPS over a time window of at least one volume scan period. So the problem is formulated as a binary genetic algorithm, and the objective of this problem is minimizing the cost function of (7).

GA operates through a simple but important iteration in four main steps [13]: the creation of population of strings, the evaluation of each string, the selection of the best or fittest 
strings and finally the genetic manipulation to create the new population of strings. The algorithm starts with initial random sets of individuals called the initial population. The objective function (7) for each individual is evaluated. On the basis of this evaluation, a new set of population is created according to three major genetic operators including selection, crossover and mutation in addition to four control parameters of population size, selection pressure, crossover rate and mutation rate. The procedure is iteratively repeated until a defined terminating condition is reached and satisfied.

\section{- Selection}

Selection is one of the three major genetic operators that chooses a chromosome from the current generation's population to be included in the population of next generation. Selection operator includes tournament, roulette wheel, top percent and best selection sections. In the tournament step of designed algorithm, a small subset of chromosomes is picked randomly and the chromosome with the lowest cost in this subset becomes a parent.

\section{- Crossover}

Crossover is an operator that combines two chromosomes to produce a new chromosome [16]. The major idea in crossover is that the child may be better than parents. The crossover operator includes one-point, two-point and uniform operation. Crossover occurs according to definable crossover probability. In the proposed GA filter, the one-point method is used and the crossover probability $\left(P_{c}\right)$ is defined as a fixed value of 0.9 .

\section{- Mutation}

Mutation is a genetic operator that alters one or more gens of a selected chromosome. Mutation points are randomly selected among the $\left(N_{\text {pop }} \times N_{\text {bits }}\right)$ bits in the population matrix. If the selected gen in a single point mutation changes from 1 to 0 or conversely, new individuals are created and added to the population. Mutation occurs during evolution according to a certain mutation probability. In the presented algorithm, the relationship between the mutation probability and the generation number is considered as follows [17].

$p_{m}=\frac{1}{240}+\frac{0.11375}{2^{t}}$

where, $p_{m}$ denotes the mutation probability and $t$ is the generation number.

Block diagram of Fig. 2 shows the main structure of the proposed GA algorithm of vertical channel filter.

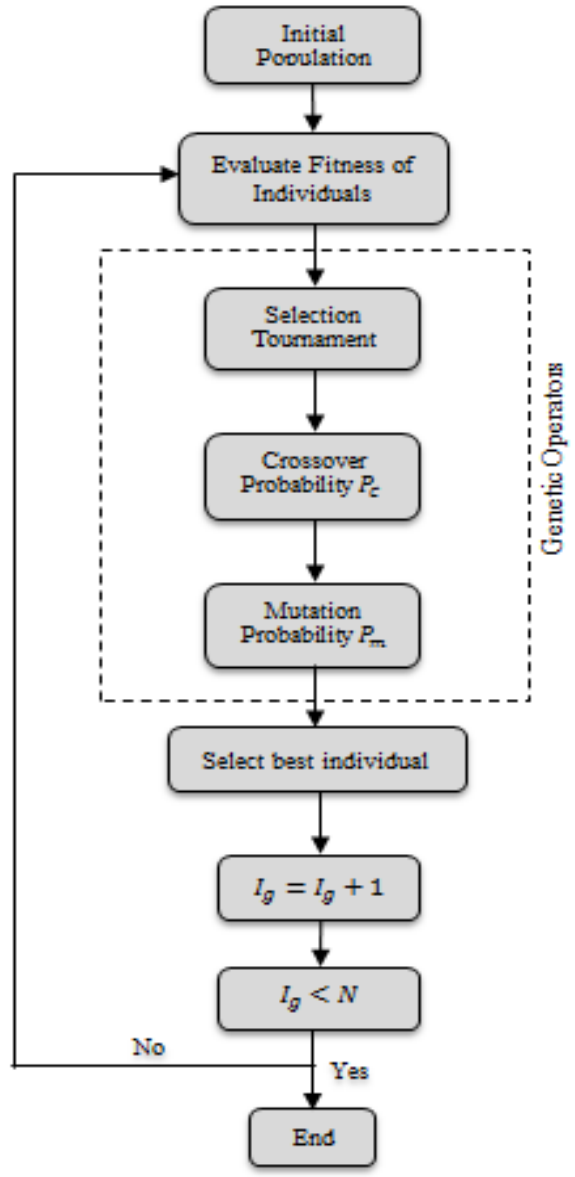

Fig.2 Block diagram of binary GA for vertical channel error compensation.

The proposed GA algorithm of vertical channel filter is summarized in five main steps as follows.

Step 1: The algorithm's parameters comprising of chromosome length, number of initial population, crossover probability $\left(P_{c}\right)$ and number of generation $(N)$ are initialized.

Step 2: For the initial population, the gains of vertical damping loop, $K_{1}, K_{2}$ and $K_{3}$ are generated.

Step 3: The objective function of (7) is evaluated for each chromosome. In this step, the best individual and also the individuals that have the least parameter distance from the best individual are reserved. The distance between the $i^{\text {th }}$ individual and the best individual is calculated as:

$d^{i}=\sum_{k=0}^{p_{n}}\left(P_{k}^{i}-P_{k}^{\max }\right)^{2}$

where, $p_{n}$ shows the total number of parameters. $P_{k}^{i}$ and $P_{k}^{\max }$ stand for the $k^{\text {th }}$ parameter of individual $i$, and the $k^{\text {th }}$ parameter of the best individual, respectively.

Step 4: The iterative generation, $I_{g}$ is set. For $I_{g}<N$, considering the crossover probability condition, crossover operation is done for two selected chromosomes and two 
children are generated. Furthermore, considering the mutation probability condition, mutation operation is done for the selected pair and another two children are generated. Then, the fitness of the new individuals is calculated and the dominant individuals of the population are updated.

Step 5: $I_{g}$ is set to $\left(I_{g}+1\right)$. If $I_{g}<N$, the algorithm is repeated from step 3. Otherwise, the process is stopped and the best solution is achieved.

Applying the presented algorithm, the feedback gains of vertical channel damping loop are determined and the true height is estimated.

\section{IMPLEMENTATION AND EESULTS}

In this section, the proposed GA filter algorithm for integrated barometric altitude is experimentally verified. Vehicular tests have been performed using "ADIS16407" IMU-barometer sensors and a "Vitans" integrated INS with a "Garmin 35" GPS [18], as shown in Fig. 3. The required temperature and raw pressure data are provided by corresponding sensors of ADIS16407 system. Considering the full scale ranges of the sensors, the pressure data are determined in the interval between $10 \mathrm{mbar}$ and $1200 \mathrm{mbar}$ and the vertical acceleration data could be measured in the interval $-10 \mathrm{~g}$ to $10 \mathrm{~g}$.

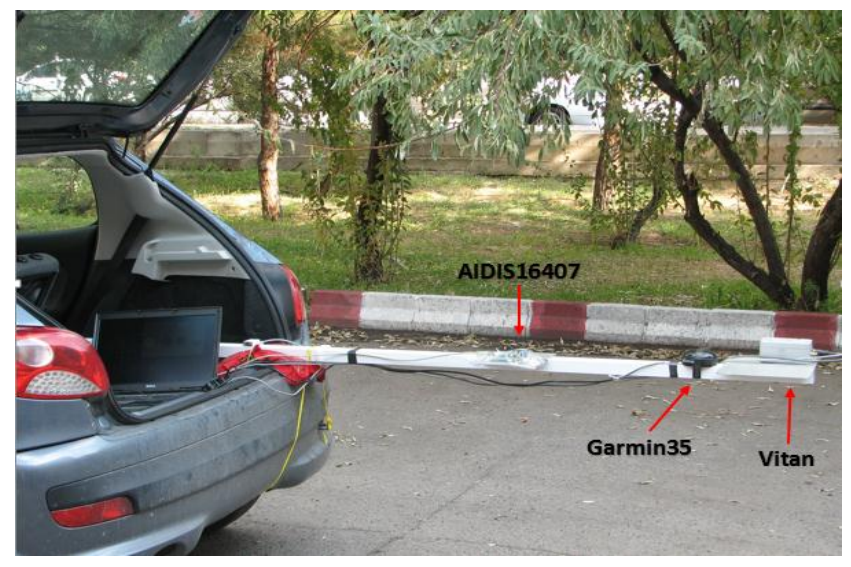

Fig.3 Block diagram of vertical channel damping loop.

Vehicular tests have been executed by experienced colleagues in navigation field. Along the mountain road tests, the vehicle's altitude is changed in large range intervals and therefore enriched calibration data are provided.

\section{A. Compensated Barometric Altitude}

In this section, the obtained altitude by the proposed compensation method of the barometric altimeter according to nonstandard atmosphere is compared with that of the traditional barometric altitude produced by Vitans system. Both the compensated barometric altitude presented in section II, and the barometric altitude of Vitans system with respect to the GPS altitude data are shown in Fig. 4.

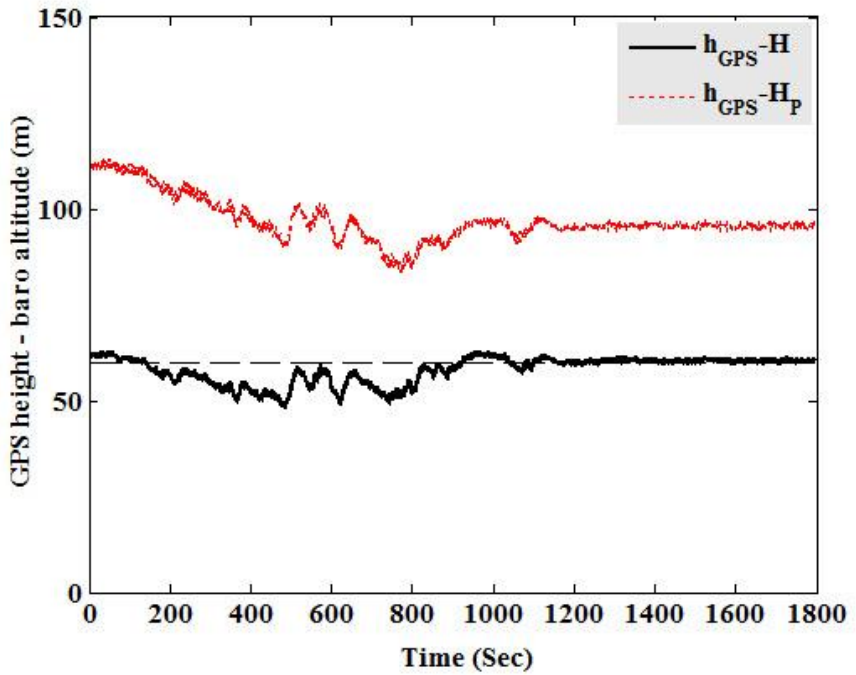

Fig.4 Compensated barometric altitude and vitans' barometric altitude with respect to GPS height.

According to Fig. 4, the error of corrected barometric altitude with respect to that of GPS is approximately $60 \mathrm{~m}$. Using WGS84 data which is the most commonly recognized model of the world geodetic system, the sea level altitude in the test region is $60 \mathrm{~m}$ above the mean sea level. Therefore, considering the fact that the GPS gives its altitude with respect to the mean-sea level, the compensated altitude of the nonstandard atmosphere is significantly accurate. Consequently, the barometric altitude correction algorithm based on nonstandard atmosphere gives near-accurate altitude in comparison to the reference GPS data.

\section{B. Implementation of $G A$}

In this section, the proposed genetic algorithm method presented in section III is experimentally verified using the vehicular test data. In the GA, random initial populations are generated for the gains of vertical damping loop. The optimal gains of the damping loop are obtained through the GA as follows.

$K_{1}=3.00$

$K_{2}=0.6452$

$K_{3}=0.8710$

By comparing the estimated height with respect to that of the GPS receiver in Fig. 5, the tracking performance of the GA based damping loop of vertical channel mechanization is assessed. The illustrated result during the vehicular test shows the valuable estimation performance of the proposed algorithm. It can be obviously inferred from Fig. 5 that the proposed GA method in the paper results in a relatively accurate estimation of vertical channel height with respect to the reference GPS height. 


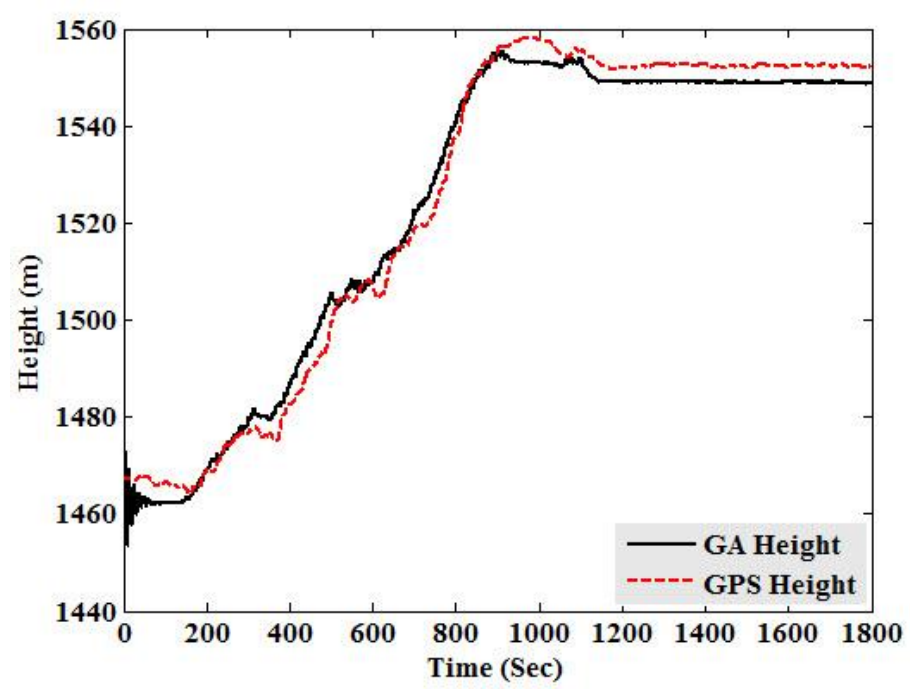

Fig.5 Estimation of vertical channel altitude for vehicular test using GA method.

\section{Comparison with Kalman Filter}

To legitimize the estimation performance of the proposed GA-based method, the vertical height is also estimated through $\mathrm{KF}$ algorithm. Using dynamical equations (6) the following dynamics of the vertical channel error is utilized in the implementation of KF [8].

$\delta \dot{V}_{Z}=2 w_{s}^{2} \delta h+A_{Z}+w(t)$

$\delta \dot{h}=\delta V_{Z}$

where, $w(t)$ represents Gaussian noise signal. Moreover, the bias of the barometer sensor is considered as a first order Gauss-Markov process modeled as [19]:

$\dot{b}(t)=-\beta b(t)+\sqrt{2 \beta \sigma^{2}} w(t)$

In (12) $\beta$ and $\sigma$ are the correlation time and the standard deviation of zero-mean white noise process, $w(t)$, respectively. The dynamical model of (11) and (12) can be rewritten in the following state space model.

$$
\dot{\mathbf{x}}=\mathbf{A} \mathbf{x}+\mathbf{B} \mathbf{u}
$$

where, the state vector, $\mathbf{x}$ and the input vector, $\mathbf{u}$ are determined as follows.

$$
\begin{aligned}
& \mathbf{x}=\left[\begin{array}{lll}
\delta V_{z} & \delta h & b
\end{array}\right]^{T} \\
& \mathbf{u}=\left[\begin{array}{ll}
A_{z} & w(t)
\end{array}\right]
\end{aligned}
$$

Using (11) and (12), the matrices A and B are obtained as:

$$
\mathbf{A}=\left[\begin{array}{ccc}
0 & 2 w_{s}^{2} & 0 \\
1 & 0 & 0 \\
0 & 0 & -\beta
\end{array}\right], \quad \mathbf{B}=\left[\begin{array}{cc}
1 & 0 \\
0 & \sqrt{2 \beta \sigma^{2}} \\
0 & 0
\end{array}\right]
$$

The following measurement equation is used in $\mathrm{KF}$ estimation algorithm.

$$
h=H+\delta h-b+v_{a}
$$

where, $v_{a}$ represents measurement noise signal. KF algorithm is imposed on the continuous-time linear dynamic system represented by (13) and the measurement (14). In Fig. 6, the performance of the GA-based vertical channel damping method is compared to the result of KF algorithm.

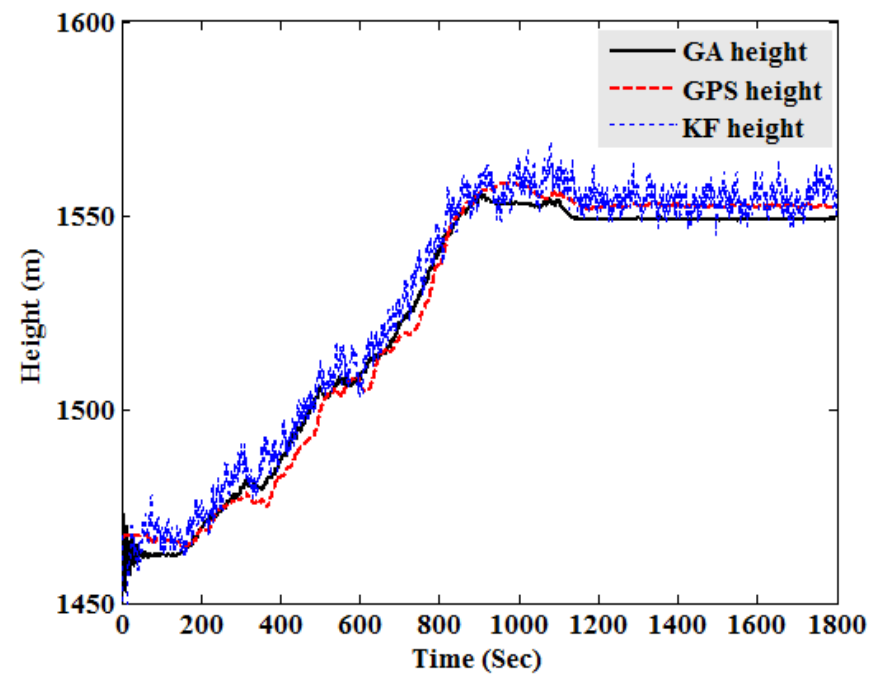

Fig.6 Comparison of GA and KF methods in estimation of vertical channel altitude.

As shown in Fig. 6, the proposed GA method yields a superior tracking compared to KF method. Furthermore, in the estimated height by the GA-based method the oscillation effect of noises has been removed, perfectly. This result becomes more significant by regarding the main advantage of GA method in which no zero-mean Gaussian noise assumption is required.

\section{V.CONCLUSION}

In many navigation applications, accurate altitude information is required. The main goal of vertical channel mechanization is to minimize the errors of vertical height and velocity. In this paper, based on binary genetic algorithm, a new algorithm has been proposed for vertical channel error compensation. vehicular test has been performed and the proposed algorithm for integrated barometric altitude was experimentally verified. The tests are executed in maneuvering conditions of the vehicle in mountain roads. It can be inferred from the results that the algorithm has a good performance for estimating the vertical channel height with an acceptable range of precision. For a fair comparison, the vertical channel height was also estimated through $\mathrm{KF}$ algorithm. The test results elucidate the superiority of GA method in tracking of accurate altitude compared to KF algorithm. Using GA method, the oscillation effect of noises on the estimated height has been removed, perfectly. Taking into account the limitation of zeromean Gaussian noise assumption in KF, the results of GA method becomes more significant. 


\section{REFERENCES}

[1] R. P. G. Collinson, Introduction to Avionics Systems, $2^{\text {nd }}$ Edition, Springer, Boston, 2003. https://doi.org/10.1007/978-1-4419-7466-2

[2] C. R. Spitzer, Avionics Development and Implementation, Taylor and Francis, Virginia, USA, 2007.

[3] S. A. A. Shahidian and H. Soltanizadeh, "Optimal Trajectories for Two UAVs in Localization of Multiple RF Sources," Trans. Inst. Meas. Control, vol. 38, 2016. pp. 908ロ916.

[4] J. Civera, A. J. Davison and J.M. Martinez, Structure from Motion Using the Extended Kalman Filter, Springer, Berlin, 2012. https://doi.org/10.1007/978-3-642-24834-4

[5] H. Nourmohammadi and J. Keighobadi, "Integration Scheme for SINS/GPS System Based on Vertical Channel Decomposition and InMotion Alignment," AUT Journal of Modeling and Simulation, DOI: 10.22060/miscj.2017.12483.5036, 2017.

[6] P. Zarchan and H. Musoff, Fundamentals of Kalman Filtering: A Practical Approach, $3^{\text {rd }}$ Edition, Progressed in Astronautics and Aeronautics, AIAA, Inc., 2009.

[7] W. S. Windall and P.K. Sinha, "Optimizing the Gains of the BaroInertial Vertical Channel," J. Guid. Control Dyn, vol. 3, 1980, pp. $172 \square 178$.

[8] J. S. Ausman, "A Kalman Filter Mechanization for the Baro-Inertial Vertical Channel," J. Inst. Navigation, vol. 1, 1991, pp. 153ロ159.

[9] J. Seo, J. G. Lee and C. G. Park, "Bias Suppression of GPS Measurement in Inertial Navigation System Vertical Channel," Proc. IEEE/ION Position, Location and Navigation Symposium, Monterey, CA, USA, April 2004.

[10] I. H. Whang, and W. S. Ra, "Barometer Error Identification Filter Design using Sigma Point Hypothese," in International Conference on Control, Automation and systems, Seoul, Korea, October 2007.

[11] J. Parviainen, J. Kantola and J. Collin, "Differential Barometry in Personal Navigation," in Proc. IEEE/ION Position, Location and Navigation Symposium, Monterey, CA, USA, May 2008. https://doi.org/10.1109/PLANS.2008.4570051

[12] A. M. Sabatini and V. Genovese, "A stochastic Approach to Noise Modeling for Barometric Altimeters," J. Sensors, vol. 13, 2013, pp. $15692 \square 15707$.

[13] M. Moness and A. M. Moustafa, "Tuning a Digital Multivariable Controller for a Lab-Scale Helicopter System Via Simulated Annealing and Evolutionary Algorithms," Trans. Inst. of Meas. Control, vol. 37, 2015, pp. 1254 $\square 1273$.

[14] Z. Jinhua, Z. Jian, D. Haifeng and W. Sun'an, "Self-Organizing Genetic Algorithm Based Tuning of PID Controllers," J. Inf. Sci, vol. 179, 2009, pp. $1007 \square 1018$.

[15] W. Wuest, Pressure and flow measurement, North Atlantic Treaty Organization, France, 1980.

[16] J. Zhang, H. S. H. Chung and W. L. Lo, "Clustering-Based Adaptive Crossover and Mutation Probabilities for Genetic Algorithms," IEEE Trans. Evol. Comput, vol. 11, 2007, pp. 326ロ335.

[17] T. C. Fogarty, "Varying the probability of mutation in the genetic algorithm," in Proc. of the $3^{\text {rd }}$ International Conference Genetic Algorithms, Morgan Kaufmann, December 1989.

[18] H. Nourmohammadi and J. Keighobadi, "Decentralized INS/GPS System with MEMS-grade Inertial Sensors Using QR-factorized CKF," IEEE Sens. J, vol. 17, 2017, pp. 3278ロ $\square 287$.

[19] H. Nourmohammadi and J. Keighobadi, "Fuzzy Adaptive Integration Scheme for Low-Cost SINS/GPS Navigation System," J. Mech. Syst. Sig. Process, vol. 99, 2018, pp. 434 $\square 449$.

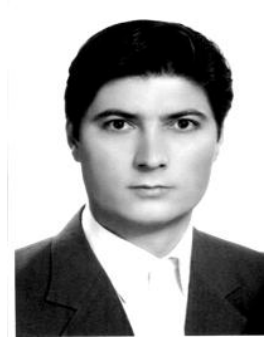

Jafar Keighobadi received the B.S. degree in Mechanical Engineering from University of Tabriz, Tabriz, Iran, in 1997, M.S. and Ph.D. degrees in Mechanical Engineering and Control Systems from Department of Mechanical Engineering, Amirkabir University of technology (Tehran Polytechnic), Tehran, Iran, in 2000 and 2008, respectively. He joined to the Faculty of Mechanical Engineering, University of Tabriz as an Assistant Professor in 2008. He is currently an Associate Professor of Mechanical Engineering Department, University of Tabriz. His research interests include artificial intelligence, estimation and identification, nonlinear robust control, and GNC.

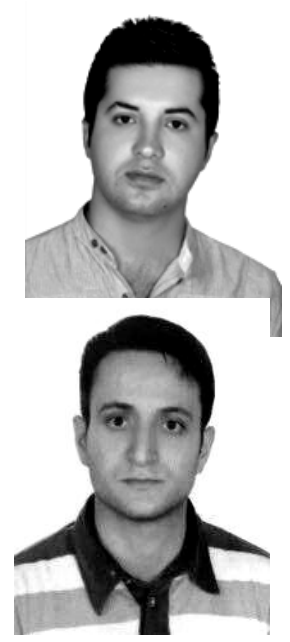

Hossein Nourmohammadi received the B.S. degree in Mechanical Engineering from Nooshirvani University of Technology, Babol, Iran, in 2010 and the M.S. degree in Mechanical Engineering from Amirkabir University of Technology (Tehran Polytechnic), Tehran, Iran, in 2012. He is currently a Ph.D. candidate in Mechanical Engineering at University of Tabriz, Iran. He has been a Research Assistant with the Navigation, Guidance and Control laboratory at University of Tabriz since 2013. His current research interests include integrated navigation systems, estimation and identification, and nonlinear adaptive control.

Sadra Rafatnia received the B.S. degree in Mechanical Engineering from Guilan University, Rasht, Iran, in 2012 and the M.S. degree in Mechanical Engineering from Tabriz University, Tabriz, Iran, in 2015. He is currently Ph.D. student in Mechanical Engineering at Sahand university of Technology (SUT). He has been a Research Assistant with the Dynamic, Vibration and Control laboratory at SUT since 2016. His current research interests include nonlinear control, estimation and optimization theory. 